\title{
Cording, a virulence-related characteristic of Mycobacteria, analysis using SEM
}

\author{
A. Sánchez-Chardi*, C. Brambilla**, E. Julián** and M. Luquin** \\ * Servei de Microscòpia, Universitat Autònoma de Barcelona (UAB), 08193, Barcelona, Spain \\ ** Dpt. de Genètica i de Microbiologia, UAB, 08193, Barcelona, Spain
}

Cord formation in Mycobacterium tuberculosis has been traditionally related to virulence since avirulent M. tuberculosis strains do not form cords. These structures consist of bacilli aligned in parallel, end to end, and side to side, along the long axis of the cord. However, similar structures were recently found in non-pathogenic and in opportunistic Mycobacterium species such as M. abscessus, M. chubuense, M. gilvum, M. haemophilum, M. marinum, M. obuense, M. parafortuitum, and M. vaccae [1-6] Although the visualization of cords has been traditionally achieved using Ziehl-Neelsen staining, the resolution of light microscopy does not allow to distinguish among cords, pseudocords, clumps or loose aggregates. Nevertheless, the observation of these formations at high resolution is crucial for two essential applications. Firstly, to identify the unknown mycobacterial components responsible for cording formation, an important step to understand virulence. Secondly, to demonstrate that cording is not specific of M. tuberculosis, since many clinical mycobacteriology laboratories base the tuberculosis diagnostic on the observation of cording morphology, and may mistakenly identify non-tuberculous mycobacteria as $M$. tuberculosis. Thus, patients would be exposed to an ineffective drug regimen with many adverse side effects. Here, we focused on sample treatment in order to certainly observe cords by using scanning electron microscopy (SEM).

The fast method of impregnation with osmium vapours was compared with the conventional method of sample treatment (chemical fixation, dehydration and critical-point drying). Both techniques require the use of spreading pellicles formed by mycobacteria grown on liquid medium, to ensure enough material to visualize this fragile structure. Osmium impregnation is an interesting method for a first approach to visualize more native structures, since mycobacteria are not immersed in liquid solutions and architectural details are preserved. As a disadvantage, bacilli are covered by an extracellular matrix making their observation difficult (Figure 1). Using the conventional method, where highly hydrophobic mycobacteria are immersed in several aqueous solutions and thereafter in alcoholic solutions, the extracellular matrix is mostly lost and bacilli are easily observed forming cords (Figures 1,2). By using the latter method we have demonstrated the formation of cords in rough M. abscessus variants, obtained from human isolates, but not in the smooth variants (Figure 2). Mycobacterium abscessus is an opportunistic emergent pathogen that causes lung infections and it is possible to misidentify it as M. tuberculosis if cording observation is used. Thus, these findings imply crucial information for clinical microbiology routine protocols since the observation of cords by light microscopy still remain as the unique method for tuberculosis diagnosis in wide developing areas. We concluded that it is necessary to stress on particular protocols to observe mycobacterial cords at ultrastructural level. Moreover, observation at ultrastructural level with SEM is the most effective method to clearly visualize cords in Mycobacterium.

\section{References}

1. Hall-Stoodley et al., FEMS Microbiology Letters 257(1): 43-49, 2006

2. Rodríguez-Güell et al., Antoine van Leeuwenhoek International Journal of General and Molecular Microbiology 90(1): 93-108, 2006

3. Julián et al., Journal of Bacteriology 192(7): 1751-1760, 2010

4. Clement et al., Journal of Clinical Microbiology 49(9): 3425-3428, 2011

5. Williams et al., Applied and Environmental Microbiology 75(7): 2091-2098, 2009

6. Sánchez-Chardi et al., Journal of Clinical Microbiology 49(6): 2293-2295, 2011 


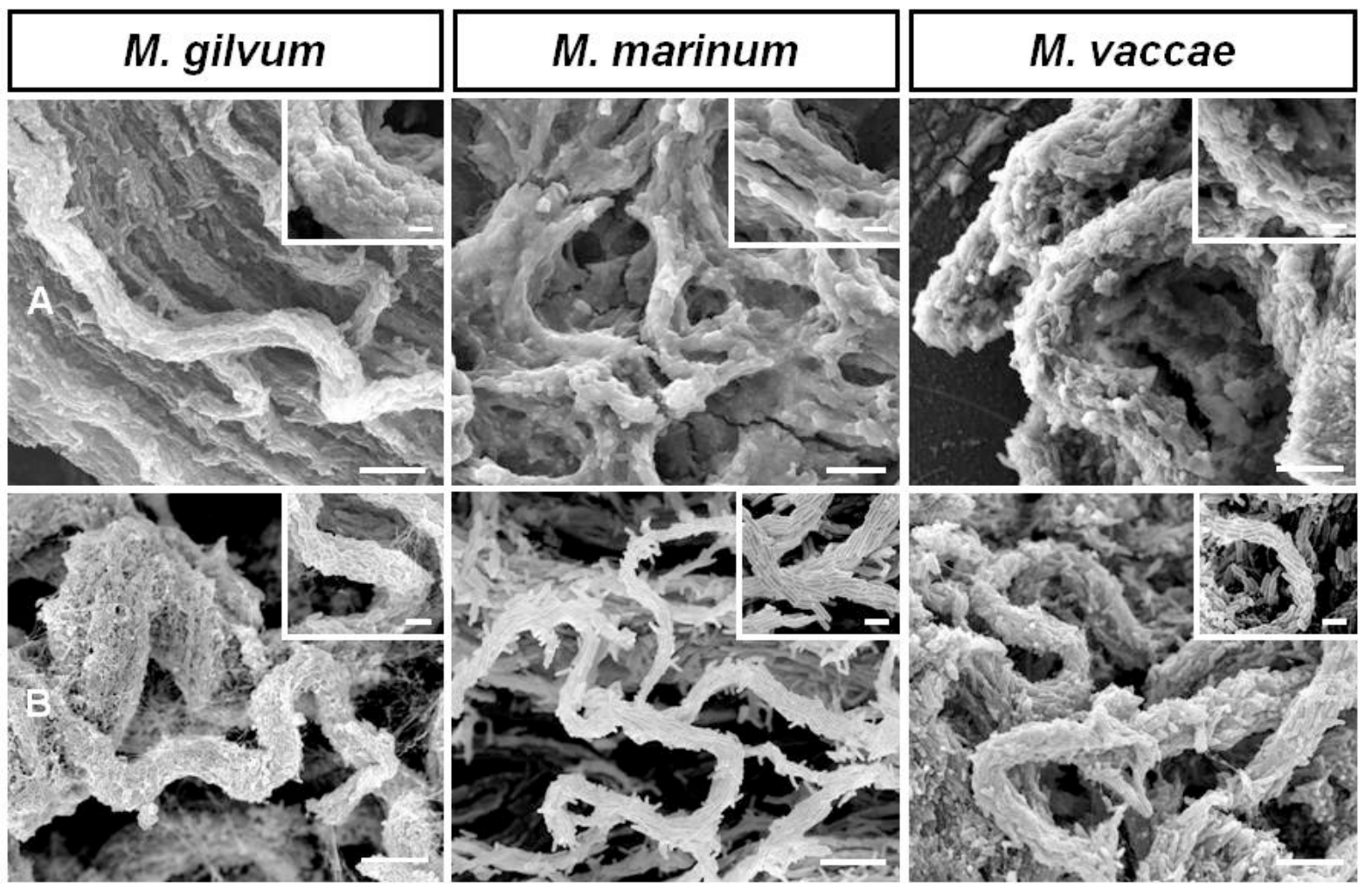

Figure 1. Representative micrographs for comparing conventional treatment (B) and osmium fixation (A) in three non-tuberculous Mycobacterium species: M. gilvum, M. marinum and M. vaccae. Bar sizes, medium magnification (large images) $7.5 \mu \mathrm{m}$ and high magnification (inserts) $1.9 \mu \mathrm{m}$.
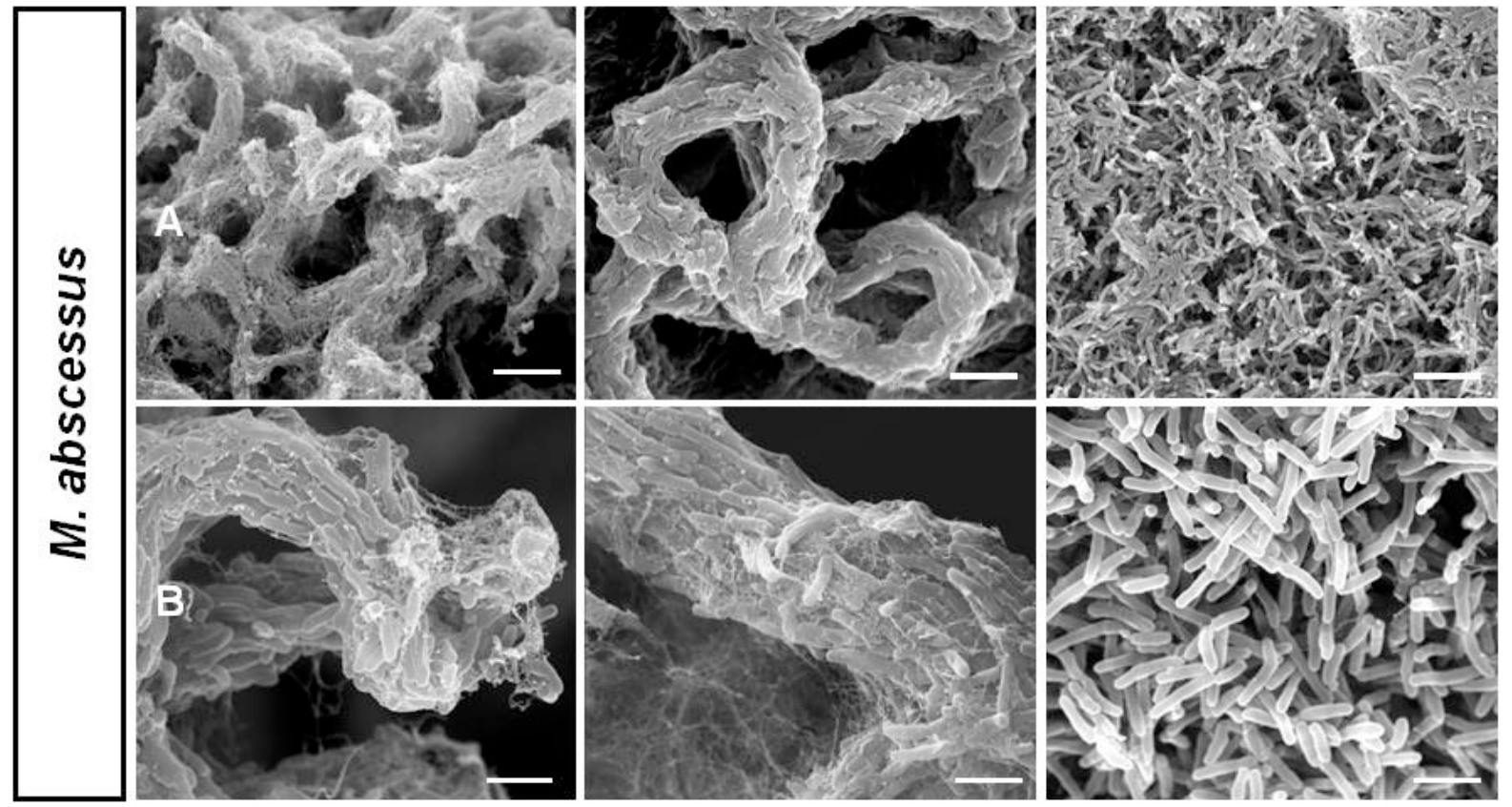

Figure 2. Representative micrographs for comparing rough and smooth strains of M. abscessus at medium (A) and high (B) magnification. Bar sizes, medium magnification $7.5 \mu \mathrm{m}$ and high magnification $1.9 \mu \mathrm{m}$. 